Objectives: With sparse data on prognostic factors in IgAV, we investigated whether pre-existing conditions are risk factors for mortality in adult IgAV patients. Methods: Observational population-based cohort study using state-wide linked longitudinal health data for adults with IgAV $(n=267)$ and matched controls $(n=1080)$ between 1980-2015. Charlson comorbidity index $(\mathrm{CCl})$ and serious infections (SI) were recorded over an extensive lookback period prior to diagnosis. Date and causes of death were extracted from the WA Death Registry. Mortality rate (deaths/1000 person-years) ratios (MRR) and time dependent survival analysis assessed the risk of death. Age and gender specific mortality rate data were obtained from the Australian Bureau of Statistics.

Results: During $9.9( \pm 9.8)$ years lookback IgAV patients accrued higher $\mathrm{CCl}$ scores $(2.60$ vs1.50 p<0.001) and had higher risk of SI (OR 8.4, p<0.001), not fully explained by $\mathrm{CCl}$ scores. During 19 years follow-up, the risk of death in IgAV patients $(n=137)$ was higher than in controls $(n=397)$ (MRR 2.06, $\mathrm{Cl} 1.70$ $2.50, \mathrm{p}<0.01$ ) and the general population (SMRR 5.64, Cl 4.25, 7.53, p<0.001). Survival in IgAV was reduced at five (72.7 vs. $89.7 \%)$ and twenty years $(45.2 \%$ vs. $65.6 \%$ ) (both $\mathrm{p}<0.05)$. $\mathrm{CCl}$ (HR1.88, $\mathrm{Cl}: 1.25$ - 2.73, $\mathrm{p}=0.001)$, renal failure (HR 1.48, Cl: $1.04-2.22, p=0.03$ ) and prior SI (HR 1.48, Cl:1.01 - 2.16, p=0.04) were independent risk factors. Death from infections (5.8 vs $1.8 \%, p=0.02)$ was significantly more frequent in IgAV patients.

Conclusion: Premorbid accrual of comorbidity is increased and predicts premature death in IgAV patients. However, comorbidity does not fully explain the increased risk of serious infections prior to diagnosis or the increased mortality due to infections in IgAV.

REFERENCES:

[1] Villatoro-Villar M, Crowson CS, Warrington KJ, Makol A, Ytterberg SR, Koster

MJ. Clinical Characteristics of Biopsy-Proven IgA Vasculitis in Children and

Adults: A Retrospective Cohort Study. Mayo Clin Proc. 2019;94(9):1769-80. Acknowledgements: The authors would like to acknowledge the support of the Arthritis Foundation of WA and acknowledge the Western Australian Data Linkage Branch, the Western Australian Department of Health, and the data custodians of, the Hospital and Morbidity Data Collection, the Emergency Department Data Collection the WA Cancer Register and the WA Death Register for their assistance with the study.

Disclosure of Interests: None declared

DOI: 10.1136/annrheumdis-2021-eular.2307

\section{AB0394 CLINICAL CHARACTERISTICS OF EOSINOPHILIC GRANULOMATOSIS WITH POLYANGIITIS: A SINGLE- CENTER RETROSPECTIVE ANALYSIS ON 52 CASES OF CHINESE PATIENTS}

L. F. Chen ${ }^{1}$, Y. Mo ${ }^{1}$, Q. H. Li ${ }^{1}$, D. H. Zheng ${ }^{1}$, L. Dai ${ }^{1}{ }^{1}$ Sun Yat-Sen Memorial Hospital, Sun Yat-Sen University, Rheumatology, Guangzhou, China

Background: Eosinophilic granulomatosis with polyangiitis (EGPA) is a rare and heterogeneous systemic vasculitis. Different patients or the same patient in different stages show different manifestations, which may lead to misdiagnosis and delay treatment.

Objectives: To analyze the clinical characteristics in Chinese patients with EGPA.

Methods: EGPA patients who fulfilled the 1990 ACR classification criteria were included between December 2003 and April 2020. The demographic and clinical characteristics were collected and analyzed retrospectively.

Results: There were 52 EGPA patients recruited, $34(65.4 \%)$ patients were males and the median age at onset was $47(38 \sim 55)$ years. The median duration from disease onset to diagnosis was 30(4 96) months. For initial symptoms, respiratory manifestations $(61.5 \%)$ were the most common, including $42.3 \%$ patients beginning with asthma, followed by $21.2 \%$ with nose/paranasal sinuses manifestations. Respiratory medicine $(53.8 \%)$ were the most common department at first visit, followed by rheumatology medicine (11.5\%, Figure $1 \mathrm{~A})$. There were $44.2 \%$ EGPA patients definitely diagnosed at the department of rheumatology or after consultation by rheumatologists.

During the whole disease process, the most common clinical manifestations were asthma (88.5\%), then nose/ paranasal sinuses (84.6\%), pulmonary $(76.9 \%)$ and nervous system $(61.5 \%)$ manifestations, followed by constitutional symptom $(44.2 \%)$, heart $(36.5 \%)$ and skin $(23.1 \%)$ involvement. Only $9.6 \%$ patients had gastrointestinal tract involvement and $3.8 \%$ had renal involvement (Figure 1B). There were $46(88.5 \%)$ patients showing the ratio of peripheral blood eosinophils $>10 \%$ at diagnosis. Among the rest 6 patients, 3 had higher eosinophil ratio before diagnosis, while the other 3 patients had been treated with glucocorticoid before diagnosis, of whom 2 patients showed pathological eosinophil infiltration in lung or paranasal sinuses mucosa, respectively. There were $8(15.4 \%)$ patients with positive ANCA. Compared with EGPA patients with negative ANCA, they had lower incidence of asthma (62.5\% vs. 93.2\%), but higher incidence of constitutional symptoms $(87.5 \%$ vs. $36.4 \%)$, arthralgia $(50.0 \%$ vs. $6.8 \%)$ and renal involvement $(25.0 \%$ vs. $0.0 \%)$, higher peripheral eosinophil count $\left[2.06(0.80 \sim 4.51) \times 10^{9} / \mathrm{L}\right.$ vs. $\left.1.81(0.93 \sim 3.32) \times 10^{9} / \mathrm{L}\right]$, ESR $[20(7 \sim 77) \mathrm{mm} / \mathrm{h}$ vs.
18(9 42) $\mathrm{mm} / \mathrm{h}$ ] and CRP [18.5(3.2 65.9) mg/L vs. 3.3(3.2 13.0) mg/L], higher Birmingham vasculitis activity score $[17(10 \sim 22)$ vs. 13(9 15)] and vasculitis damage index $[3(1 \sim 3)$ vs. 2(1 2), all $P<0.05]$. There were $21.2 \%$ EGPA patients showing poor prognostic factors according to five-factor score (FFS) and $34.6 \%$ patients according to the revised FFS.

Conclusion: EGPA patients may have no asthma especially those with positive ANCA. Multi-disciplinary collaboration especially based on rheumatologists and pulmonologists should be emphasized for early identification and prompt treatment.
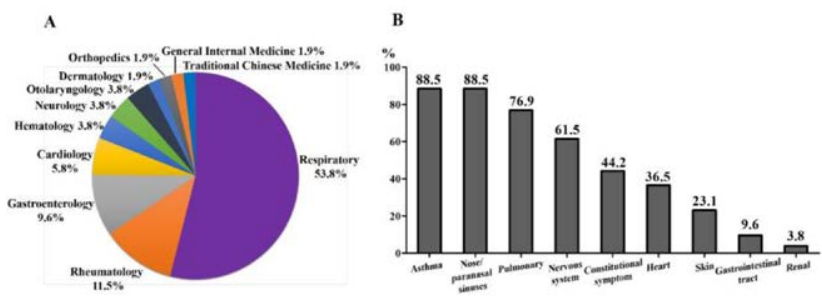

Figure 1. Department at first visit (A) and clinical manifestations during the whole disease process of 52 Chinese EGPA patients(B)

Disclosure of Interests: None declared

DOI: 10.1136/annrheumdis-2021-eular.2359

\section{AB0395 RENAL INVOLVEMENT IN HENOCH-SCHÖNLEIN PURPURA}

N. Ben Chekaya ${ }^{1}$, S. Daada ${ }^{1}$, M. Brahim ${ }^{1}$, I. Chaabene ${ }^{1}$, R. Klii ${ }^{1}$, M. Kechida ${ }^{1}$ S. Hammami ${ }^{1}{ }^{1}$ Fattouma Bourguiba Hospital, Internal Medicine and Endocrinology Departement, Monastir, Tunisia

Background: Henoch-Schönlein purpura (HSP), also known as rheumatic purpura, is an immune complex vasculitis affecting small vessels with dominant IgA deposits. Clinical manifestations mainly involve cutaneous purpura, arthralgias and/or arthritis, acute enteritis and glomerulonephritis. HSP is more common among children than adults. Kidney damage is the principal prognostic determinant in HSP. The aim of this study is to evaluate the renal manifestations of HSP.

Objectives: The aim of this study is to evaluate the renal manifestations of HSP.

Methods: This was a retrospective study of 35 patients with diagnosed HSA who were admitted between the time period may 2008 and november 2018. All of these patients met the American College of Rheumatology classification criteria for a diagnosis of HSP. Renal involvement was defined as the presence of proteinuria or hematuria.

Results: The study group consisted of 35 patients, 20 women (57\%) and 15 men (43\%). The mean age was $48.8 \pm 18.7$ years. The incidence rates of skin, joint, gastrointestinal and central nervous system involvement were 91.4, 77.1 54.2 and $2.8 \%$, respectively. Elevated serum IgA levels occurred in $5.7 \%$. Six patients $(17.1 \%)$ were given corticosteroids during the first 3 months of their HSP, which were indicated for severe gastrointestinal $(n=5)$ or renal involvement $(n=1)$. Eighteen of the thirty five patients $(51.42 \%)$ had renal involve ment. There were 12 women and six men. All patients had proteinuria (100\%). Hematuria has been identified in 11 patients $(61.1 \%)$ and renal insufficiency in 4 patients $(22.2 \%)$. The renal involvement was mainly detected in the first month. Renal biopsy was affected in four patients (22.2\%). An endocapillary glomerulonephritis was found in 3 patients and an extra capillary necrotizing glomerulonephritis in one patient. The immunofluorescence showed the presence of IgA deposits in 4 patients.

Conclusion: Renal involvement dominates the prognosis for the disease. The most common clinical expression is the association of microscopic hematuria with proteinuria.

Disclosure of Interests: None declared

DOI: 10.1136/annrheumdis-2021-eular.2670

\section{AB0396 RAPID IMPROVEMENT IN CYSTOID MACULAR EDEMA WITH HIGH DOSE INTRAVENOUS METHYLPREDNISOLONE IN NON-INFECTIOUS UVEITIS OF DIFFERENT IMMUNE MEDIATED INFLAMMATORY DISEASES}

N. Vegas-Revenga ${ }^{1}$, J. L. Martín-Varillas ${ }^{2}$, V. Calvo-Río ${ }^{3}$, I. González-Mazón ${ }^{3}$ L. Sanchez-Bilbao ${ }^{3}$, E. Beltrán ${ }^{4}$, A. Fonollosa ${ }^{5}$, O. Maiz-Alonso ${ }^{6}$, A. Blanco ${ }^{7}$, M. Cordero-Coma ${ }^{8}$, N. Ortego ${ }^{9}$, I. Torre-Salaberri ${ }^{10}$, F. Francisco ${ }^{11}$, S. Muñoz Fernandez $^{12}$, M. D. M. Esteban-Ortega ${ }^{13}$, M. Díaz-Llopis ${ }^{14}$, J. Cañal $^{15}, \mathrm{~J}$ A. Ventosa ${ }^{15}$, R. Demetrio-Pablo ${ }^{15}$, L. Domínguez ${ }^{16}$, M. Agudo-Bilbao ${ }^{3}$, 